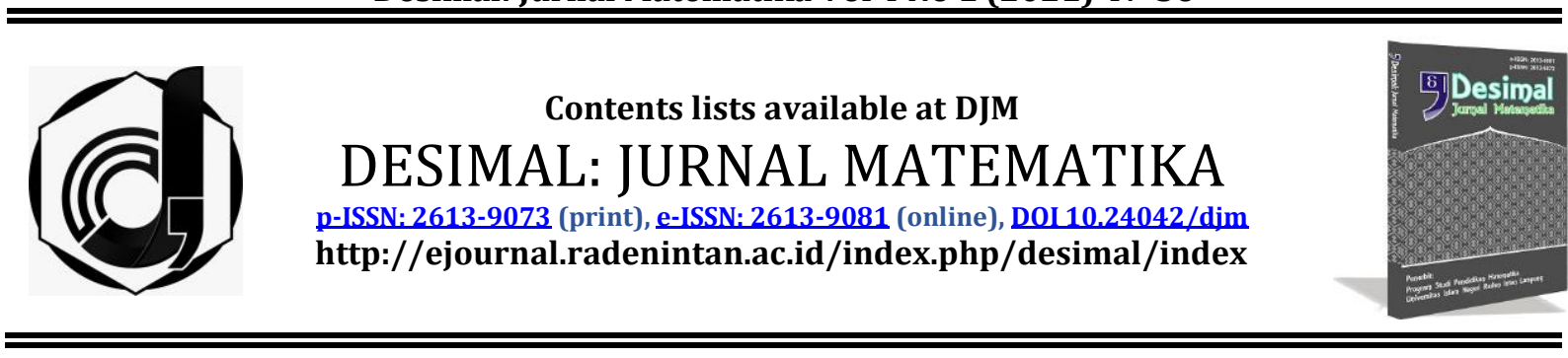

\title{
The analysis of the sensitivity of vaccination parameters in a tuberculosis transmission model
}

\author{
S. L. Chasanah*, P. Ferdias, D. E. Nurvazly \\ Universitas Lampung, Indonesia
}

\begin{tabular}{|c|c|}
\hline ARTICI & LE INFO \\
\hline \multicolumn{2}{|c|}{ Article History } \\
\hline Received & : 10-09-2020 \\
\hline Revised & $: 17-12-2020$ \\
\hline Accepted & $: 18-01-2021$ \\
\hline Published & :25-01-2021 \\
\hline \multicolumn{2}{|l|}{ Keywords: } \\
\hline \multicolumn{2}{|c|}{ Vaccination Parameters; } \\
\hline \multicolumn{2}{|c|}{ Tuberculosis; Transmission Model. } \\
\hline \multicolumn{2}{|c|}{$\begin{array}{l}\text { *Correspondence: E-mail: } \\
\text { siti.chasanah@fmipa.unila.ac.id }\end{array}$} \\
\hline \multicolumn{2}{|l|}{ Doi: } \\
\hline \multicolumn{2}{|c|}{$10.24042 /$ djm.v4i1.7116 } \\
\hline
\end{tabular}

\begin{abstract}
Tuberculosis (TB) is one of the most dangerous infectious diseases that caused 1,5 million people died from TB in 2018 (including 251.000 people with HIV). As the preventive strategy for the spread of $T B$, we can use BCG vaccination. This study aims to investigate and analyze the sensitivity of vaccination parameters (to newborns and adults). This model divided the human population into five classes: susceptible $(S)$, vaccinated $(V)$, high-risk $(E)$ and lowrisk $(L)$ latent, and infectious (I). Analysis of the mathematical model was discussed by finding the existence and analyzing the model equilibrium's stability based on the Basic Reproduction Number $\left(\mathcal{R}_{0}\right)$. Furthermore, we determined the sensitivity analysis of the proportion of vaccine and other parameters that affect the TB transmission model. The numerical experiment shows that vaccination to adults more effective than newborns.

http://ejournal.radenintan.ac.id/index.php/desimal/index
\end{abstract}

\section{INTRODUCTION}

Tuberculosis (TB) is one of the most dangerous infectious diseases that caused many death cases worldwide. It is caused by Mycobacterium tuberculosis and attacks the human respiratory system. There are 1,5 million people died from TB in 2018 (including 251.000 people with HIV). In the same year, 10 million people infected with TB worldwide, with 5,7 million men, 3,2 million women, and 1,1 million children (World Health Organization (WHO), 2019). The number of deaths that occurred due to the spread of TB that uses air as a medium. When infectious people with TB coughing or sneezing, the TB bacteria in the sputum splash will spread into the air until inhaled by healthy people.

People with TB cannot directly transmit TB bacteria to other individuals (infectious). There is an incubation period to be an active TB, called TB latent infection. The length of time from latent TB to active TB depends on each individual's immunity (Grange et al., 2011). When the immunity is low, the time needed to become infectious is faster than individuals with high immunity. People with latent TB infection have no symptoms, don't feel sick, and can't spread 
TB bacteria to others. But they usually have positive TB skin tests and may develop active TB if they don't receive latent TB treatment (Centers for Disease Control and Prevention (CDC), 2020). The large population and high proportion of infected increased the number of people with latent TB. Around $80 \%$ of the number of latent TB is in the WHO Southeast Asia, Western Pacific, and Africa region, compared to $65 \%$ of the total population (Houben \& Dodd, 2016). The fact that the bacteria cannot be directly detected in vivo during latent makes an important hurdle in TB research (Petruccioli et al., 2016).

The government uses Bacillus Calmette-Guerin (BCG) vaccines as a preventive strategy for the spread of TB. On the other hand, the BCG vaccine is unable to protect a person from TB disease forever. The BCG vaccine protection will begin to decrease after $10-15$ years of vaccine administration (Nguipdop-Djomo et al., 2016). The BCG vaccine is also cannot protect individuals in total. Vaccinated individuals can still be infected with TB disease. The BCG vaccine can only provide about $80 \%$ protection against children and $50 \%$ protection against adults (Department of Health and Human Services, 2016).

In general, only newborns were getting a vaccine. However, the children and adults who have never been vaccinated can also get vaccines (Department of Health and Human Services, 2016). In other words, vaccinated individuals can't get the vaccine again. Besides that, individuals with positive TB also cannot get the vaccine. People do a Mantoux skin test (TST) before vaccinated. If TST results are positive, then they cannot be vaccinated but treated. Conversely, if an individual was a negative TB (TST result is negative), they can be given a vaccine.

The researchers try to suppress the spread of TB by using the mathematical model. It can be useful to analyze the spread and control of infectious diseases (Hethcote, 2000). This result can represent the spread of TB in real life. There is much research about optimal control of TB disease, such as in the dynamic model of smoking TB transmission(Choi et al., 2015), mitigating (Kim et al., 2018), vaccination, and treatment (Yang et al., 2016). In Nyabadza \& Kgosimore (2012) modeled the spread of TB disease in the population of children and adults. The following year, Mishra \& Srivastava (2014) constructed a model of disease spread in lung TB and multidrugresistant patients with vaccination. Using a mathematical model, there was estimated that approximately 1,7 billion individuals were latently TB globally (Houben \& Dodd, 2016). In Yang et al. (2016) created a TB transmission model using vaccination and treatment. Subsequently, Siyu et al. also researched the TB deployment model using a mixed vaccination strategy (Liu et al., 2017). Kim et al. divide the population into four groups: susceptible, high-risk latent, lowrisk latent, and infectious (Kim et al., 2018). Based on the above exposure, the study aims to investigate and analyze the sensitivity of vaccination parameters (to newborns and adults).

There are five sections in this paper. In section 2, we describe the method to construct the TB transmission model. Then, we analyzed the model in Section 3 and the numerical experiment in Section 4. Finally, we conclude our paper in Section 5.

\section{METHOD}

In this section, we describe variables and parameters for the model. Then, we give assumptions on the TB transmission model. Based on that, we construct the TB transmission process and the dynamic model of TB transmission. 


\subsection{Model formulation}

In this paper, we construct the TB transmission model with vaccination strategies. Let us assume that the population is closed and constant. There is no migration, and birth has the same number as death. The population is homogeneous, which means that each individual has the same probability of having contact with another individual; the recovery rate is the same unless otherwise stated. We divided the population into five classes, i.e., vaccinated $(V)$, susceptible $(S)$, high-risk latent $(E)$, low-risk latent $(L)$, and infectious $(I)$. Total population denote with $N$, i.e., $N(t)=V(t)+S(t)+E(t)+L(t)+I(t)$.

In this model, the vaccine was given to newborns and adults who have never been vaccinated or infected with TB. When newborns and adults were vaccinated, they will move to a vaccinated compartment $(V)$. Otherwise, they will become susceptible individuals $(S)$.

Furthermore, the BCG vaccine does not always protect humans from TB (Centers for Disease Control and Prevention (CDC), 2020; Grange et al.,
2011; World Health Organization (WHO), 2019). The BCG vaccine can only provide about $80 \%$ protection to children and $50 \%$ protection to adults. The effectiveness of this vaccine depends on each individual's condition. The percentage of vaccines that can't protect humans denoted with $\xi$. During the incubation period (exposed), the individual population will also be divided into high-risk and low-risk latent. Low-risk latent individuals can't be infectious to others, even though they have M. tuberculosis in their bodies. People with TB will move to the high-risk latent class $(E)$. If people with high-risk latent TB have low immune and don't get treatment, they will become infectious TB (I) with a progression rate $\kappa$. Otherwise, if they have high immune, they become lowrisk latent individuals $(L)$ with a progression rate $\alpha$. Infected person cannot completely recover from TB disease. Even if an infected person has completed treatment, there are still TB bacteria in his body. Therefore, the individual will move to a low-risk latent group. The parameter definition for a model is given in Table 1 .

Table 1. Definition and Values of Parameters for The Model

\begin{tabular}{|c|c|c|c|c|}
\hline Symbol & Definition & Unit & Value & References \\
\hline$N$ & Total population & human & 1000 & Assumed \\
\hline$b$ & Natural birth rate & $1 /$ year & $1 / 70$ & Assumed \\
\hline$\mu$ & Natural death rate & $1 /$ year & $1 / 70$ & Assumed \\
\hline$u_{1}$ & $\begin{array}{l}\text { Proportion of newborns that } \\
\text { vaccinated }\end{array}$ & - & {$[0,1]$} & Assumed \\
\hline$u_{2}$ & $\begin{array}{l}\text { Proportion of individuals that } \\
\text { never vaccinated }\end{array}$ & - & {$[0,1]$} & Assumed \\
\hline$\beta$ & Transmission rate & 1/human. year & 11,7345 & (Kim et al., 2018) \\
\hline$\xi$ & $\begin{array}{l}\text { Percentage of vaccine cannot } \\
\text { protect human }\end{array}$ & - & {$[0,1]$} & Assumed \\
\hline$\alpha$ & Progression rate from $E$ to $L$ & $1 /$ year & 0,2077 & (Kim et al., 2018) \\
\hline$\kappa$ & Progression rate from $E$ to $I$ & $1 /$ year & 0,0298 & (Kim et al., 2018) \\
\hline$\gamma$ & Treatment rate & $1 /$ year & 0,2906 & (Kim et al., 2018) \\
\hline$p$ & $\begin{array}{l}\text { Proportion of treatment failure } \\
\text { probability }\end{array}$ & - & {$[0,1]$} & Assumed \\
\hline
\end{tabular}

2.2 Dynamic model of TB transmission

Figure 1. is a flow diagram of the TB transmission model in a population. From the assumption and TB transmission process, we formulated a TB model given by the TB dynamic system (1). The definition and range of the parameter used for carrying out numerical simulations of a model (1) are lists in Table 1. 
Desimal, 4 (1), 2021 - 50

S. L. Chasanah, P. Ferdias, D. E. Nurvazly

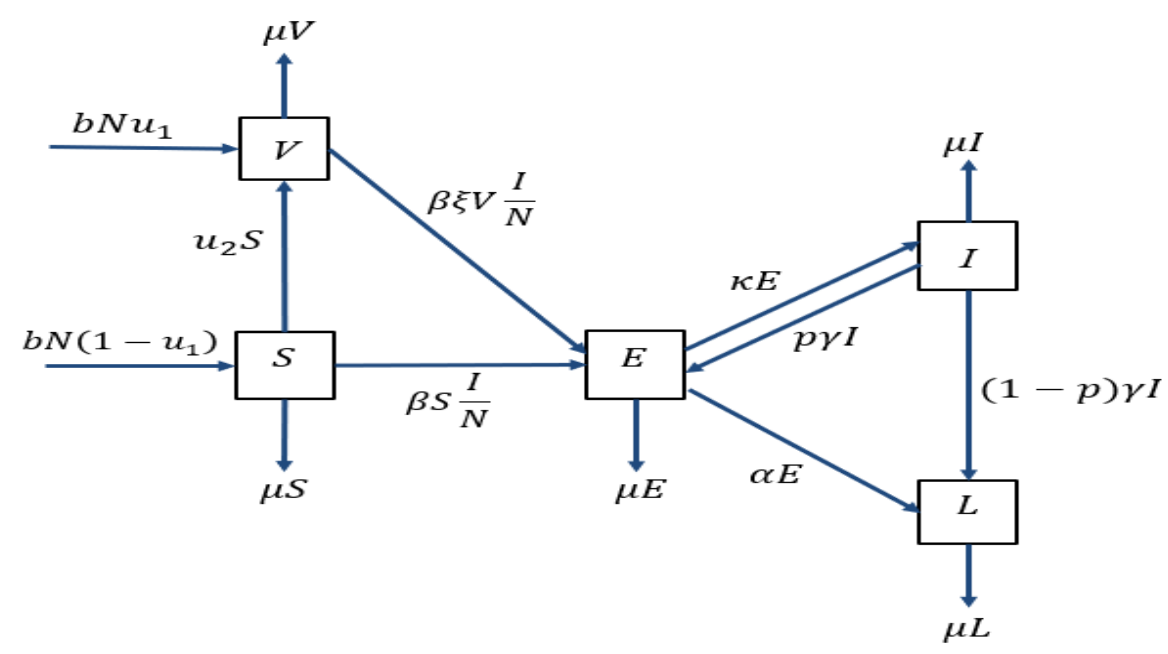

Figure 1. TB Transmission Process

$$
\begin{array}{cc}
\frac{d S(t)}{d t}= & b N(t)\left(1-u_{1}\right)-u_{2} S(t) \\
& -\frac{\beta S(t) I(t)}{N(t)} \\
& -\mu S(t), \\
\frac{d V(t)}{d t}= & b N(t) u_{1}+u_{2} S(t)-\frac{\beta \xi V(t) I(t)}{N(t)}- \\
\mu V(t), & \quad+p \gamma I(t) \\
\frac{d E(t)}{d t}= & \frac{\beta \xi V(t) I(t)}{N(t)}+\frac{\beta S(t) I(t)}{N(t)} \\
& -\kappa E(t)-\alpha E(t)-\mu E(t), \\
\frac{d L(t)}{d t}=\alpha E(t)+(1-p) \gamma I(t)-\mu L(t), \\
\frac{d I(t)}{d t}=\alpha E(t)-\gamma I(t)-\mu I(t) .
\end{array}
$$

\section{ANALYSIS OF THE MODEL}

In this section, we analyzed the equilibrium point's existence and calculated the Basic Reproduction Number $\left(R_{0}\right)$. Using that equilibrium point, we analyzed the local stability criteria of the model.

3.1 Existence of the equilibria and calculation of the Basic Reproduction Number $\left(\mathcal{R}_{0}\right)$

The equilibrium is a condition where the system will not change. This point is used to understand the condition of the system at $t \rightarrow \infty$ and obtained by finding the solution of the equation $\frac{d S_{1}}{d t}=\frac{d S_{2}}{d t}=$ $\frac{d V}{d t}=\frac{d L}{d t}=\frac{d E}{d t}=\frac{d I}{d t}=\frac{d R}{d t}=0$. There are two equilibrium points of the system (1), disease-free equilibrium and endemic equilibrium. By determining the solution of model (1) and substituting $I=0$, we obtain disease-free equilibrium below.

$$
\begin{aligned}
E_{0} & =\left(S_{0}, V_{0}, E_{0}, L_{0}, I_{0}\right) \\
& =\left(\frac{b N\left(1-u_{1}\right)}{\mu+u_{2}}, \frac{b N\left(\mu u_{1}+u_{2}\right)}{\left(\mu+u_{2}\right) \mu}, 0,0,0\right)
\end{aligned}
$$
Moreover, the model (1) has the endemic equilibria $E_{1}=\left(S_{1}, V_{1}, E_{1}, L_{1}, I_{1}\right)$, where:

$$
\begin{aligned}
& S_{1}=\frac{b N^{2}\left(1-u_{1}\right)}{\left(\mu+u_{2}\right) N+I_{1} \beta^{\prime}} \\
& V_{1}=\frac{b\left(\left(\mu u_{1}+u_{2}\right) N+u_{1} \beta I_{1}\right) N^{2}}{\left(\xi \beta I_{1}+\mu N\right)\left(\left(\mu+u_{2}\right) N+\beta I_{1}\right)^{\prime}} \\
& E_{1}=\frac{(\gamma+\mu) I_{1}}{\kappa}, \\
& L_{1}=\frac{((1-p) \gamma \kappa+\alpha(\gamma+\mu)) I_{1}}{\kappa \mu},
\end{aligned}
$$$$
I_{1}=I_{1} \text {. }
$$

The basic reproduction number $\left(\mathcal{R}_{0}\right)$ is the average occurrence of new cases by one infected individual in a population (Diekmann et al., 2010). $\mathcal{R}_{0}$ is often considered a threshold value that determines whether a disease becomes endemic or not. According to Van Den Driessche \& Watmough (2002), there are several possibilities $\mathcal{R}_{0}$. If $\mathcal{R}_{0}<1$, the infectious TB can only spread to an average of less than one person, then the TB disease will not spread. Otherwise, if $\mathcal{R}_{0}>1$, the infectious TB can spread to an average of more than one person, then the disease will spread.

In our model, we use the nextgeneration matrix, denoted by $K$, 
approach to compute $\mathcal{R}_{0}$. Firstly, we linearized the infectious subsystem with the Jacobian matrix. Then, decomposed the Jacobian matrix to the transmission matrix $T$ and transition matrix $\Sigma$ so that $T+\Sigma$. In model (1), there are three infectious states $(E, L$, and $I)$ and two uninfected states ( $V$ and $S$ ). from the linearized infection subsystem, we obtain the transmission matrix and transition material are, respectively,

$$
\begin{aligned}
T & =\left[\begin{array}{ccc}
0 & 0 & \frac{\xi \beta V}{N}+\frac{\beta S}{N} \\
0 & 0 & 0 \\
0 & 0 & 0
\end{array}\right], \\
\Sigma & =\left[\begin{array}{ccc}
-\alpha-\kappa-\mu & 0 & p \gamma \\
\alpha & -\mu & (1-p) \gamma \\
\kappa & 0 & -\gamma-\mu
\end{array}\right] .
\end{aligned}
$$

There are zero rows in the transmission matrix $T$ and the determinant of the matrix $K=$ $-E_{1}^{\prime} T \Sigma^{-1} E_{1}=0$. Then, we use NGM with a small domain $K_{S}$ to compute $\mathcal{R}_{0}$. To construct matrix $K_{S}$, we define matrix $M$ whose rows are linearly independent vectors spanning the rows $T$, and matrix $C$ whose columns are linearly independent vectors spanning the columns of $T$, so that $T=C M$.

$M=\left[\begin{array}{lll}0 & 0 & 1\end{array}\right], \quad C=\left[\begin{array}{c}\frac{\xi \beta V}{N}+\frac{\beta S}{N} \\ 0 \\ 0\end{array}\right]$

The NGM with small domain is given by,

$$
\begin{aligned}
K_{S}= & -M \Sigma^{-1} C \\
= & \\
& {\left[\frac { \kappa } { ( \mu + ( 1 - p ) \kappa + \alpha ) \gamma + \mu ( \mu + \alpha + \kappa ) } \left(\frac{b \xi \beta\left(\mu u_{1}+u_{2}\right)}{\left(\mu+u_{2}\right) \mu}+\right.\right.} \\
& \left.\left.\frac{b \beta\left(1-u_{1}\right)}{\mu+u_{2}}\right)\right] .
\end{aligned}
$$

By finding the dominant eigenvalue of matrix $K_{S}$, we get $\mathcal{R}_{0}$ below.

$$
\begin{aligned}
\mathcal{R}_{0}= & \frac{\kappa}{(\mu+(1-p) \kappa+\alpha) \gamma+\mu(\mu+\alpha+\kappa)} . \\
& \left(\frac{b \xi \beta\left(\mu u_{1}+u_{2}\right)}{\left(\mu+u_{2}\right) \mu}+\frac{b \beta\left(1-u_{1}\right)}{\mu+u_{2}}\right)
\end{aligned}
$$

3.2 Local stability criteria of the equilibria

The equilibrium point's stability can be determined based on the sign of the eigenvalues of the Jacobian matrix $(U)$, which is evaluated at the equilibrium point. From model (1), we obtained matrix Jacobian with ordo $5 \times 5$ :

$$
\begin{aligned}
& J=\left[\begin{array}{ll}
A & B \\
C & D
\end{array}\right] \\
& \text { with }=\left[\begin{array}{ccc}
-\frac{\xi \beta I}{N}-\mu & u_{2} & 0 \\
0 & -u_{2}-\frac{\beta I}{N}-\mu & 0 \\
\frac{\xi \beta I}{N} & \frac{\beta I}{N} & -\alpha-\kappa-\mu
\end{array}\right] \\
& B=\left[\begin{array}{cc}
0 & -\frac{\xi \beta V}{N} \\
0 & -\frac{\beta S}{N} \\
0 & \frac{\xi \beta V}{N}+\frac{\beta S}{N}+p \gamma
\end{array}\right], \\
& C=\left[\begin{array}{lll}
0 & 0 & \alpha \\
0 & 0 & \kappa
\end{array}\right], \\
& D=\left[\begin{array}{cc}
-\mu & (1-p) \gamma \\
0 & -\gamma-\mu
\end{array}\right] .
\end{aligned}
$$

From matrix (5), we obtained the characteristic polynomial of degree 5 in (6).

$$
\begin{gathered}
(\lambda+\mu)^{2}\left(\lambda+\mu+u_{2}\right)\left(\sum_{i=0}^{2} a_{i} \lambda^{2-1}\right) \\
=0,
\end{gathered}
$$

with

$a_{0}=1$,

$a_{1}=\gamma+\alpha+\kappa+2 \mu$

$a_{2}=\frac{(((1-p) \gamma+\mu) \kappa+(\gamma+\mu)(\mu+\alpha)) N-\kappa \beta(\xi V+S)}{N}$.

According to Brauer \& CastilloChavezCarlos (2012), the equilibrium point is asymptotically stable if the real part of all eigenvalues of the J matrix is negative. From the characteristic polynomial (6), we obtained the eigenvalues of matrix Jacobian (5) are $-\mu$, $-\mu,-\mu-u_{2}$. To analyze the other eigenvalues, we use the Routh-Hurwitz criteria of two-degree polynomials. Based on the Routh-Hurwitz criteria. The polynomial characteristic of two-degree polynomials will obtain all negative eigenvalues if $a_{1}>0$ and $a_{2}>0$. Note that, $\quad a_{1}=\gamma+\alpha+\kappa+2 \mu>0$. To guarantee $a_{2}>0$ will be positive, it should satisfy the following condition below. 


$$
\frac{(((1-p) \gamma+\mu) \kappa+(\gamma+\mu)(\mu+\alpha)) N}{\kappa \beta(\xi V+S)}
$$

Because of the model (1) has three negative eigenvalues and the RouthHurwitz stability criteria in the polynomial (6) is satisfied, the diseasefree equilibrium is locally stable.

Local stability analysis of endemic equilibrium points was performed by analyzing the Jacobian matrix at its endemic points $(E E)$. Due to the complexity of the system, to analyze the stability of endemic equilibrium is done numerically using the parameters in Table 1., $\xi=0,2, p=0,2, u_{1}=0,2$, and $u_{2}=0.1$ until endemic equilibrium is obtained $(E E=(86,91,651,46,15,21,244,95$, 1,47)) such that $\mathcal{R}_{0}=1,29>1$.

From the Jacobian matrix, eigenvalues are obtained $\lambda_{1}=-0.01$, $\lambda_{2}=-0.56, \lambda_{3}=-0.12, \lambda_{4}=-0.01+$ $0.02 I, \lambda_{5}=-0.01-0.02 I$. Since the real part of all eigenvalues of the Jacobian matrix is negative, system (1) is locally stable in endemic conditions.

\section{NUMERICAL EXPERIMENT}

In numerical simulations, we discuss the sensitivity analysis of the parameters of the proportion of newborns and adults who were given vaccines $\left(u_{1}\right.$ and $\left.u_{2}\right)$ and the percentage of vaccines that did not protect humans $(\xi)$ to $\mathcal{R}_{0}$. In addition, we also discuss the autonomous system with various scenarios.

4.1 Sensitivity of vaccination and vaccine efficacy parameters to $\mathcal{R}_{0}$

In Figure 2, we assumed that the percentage vaccine could not protect humans $(\xi)$ is $20 \%$. When the vaccine is only given to newborns, $97,8 \%$ of newborns in the population must be vaccinated. Otherwise, if only adults that can be vaccinated, we must use a $65 \%$ vaccination proportion to reach diseasefree.

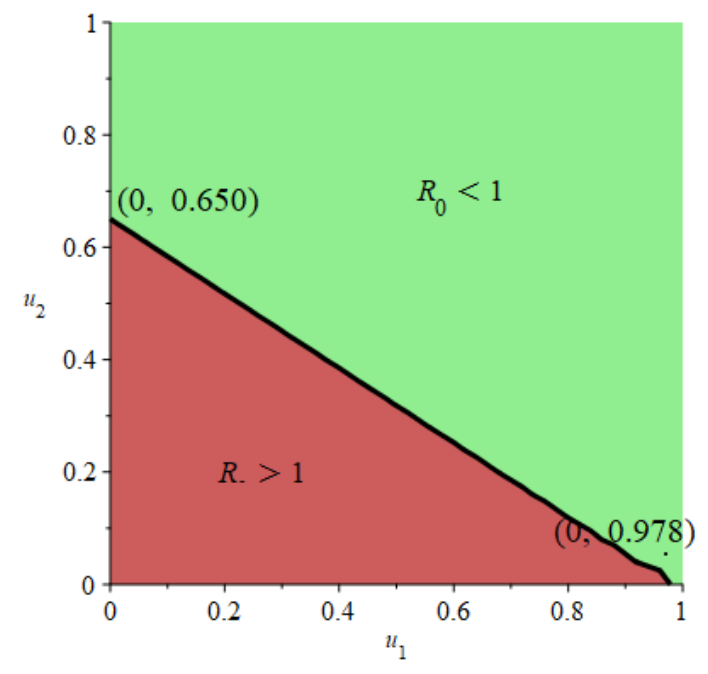

Figure 2. Sensitivity of vaccination parameters $u_{1}$ and $u_{2}$ to $\mathcal{R}_{0}$.

In Figure 3, we assume that the vaccine is only given to newborns. It appears that the lower percentage vaccine cannot protect human, the population will lead to disease-free. The minimum proportion of vaccine efficacy cannot protect humans must be less than $21,8 \%$. If vaccine have no protection, $78,3 \%$ of newborns in the population must be vaccinated.

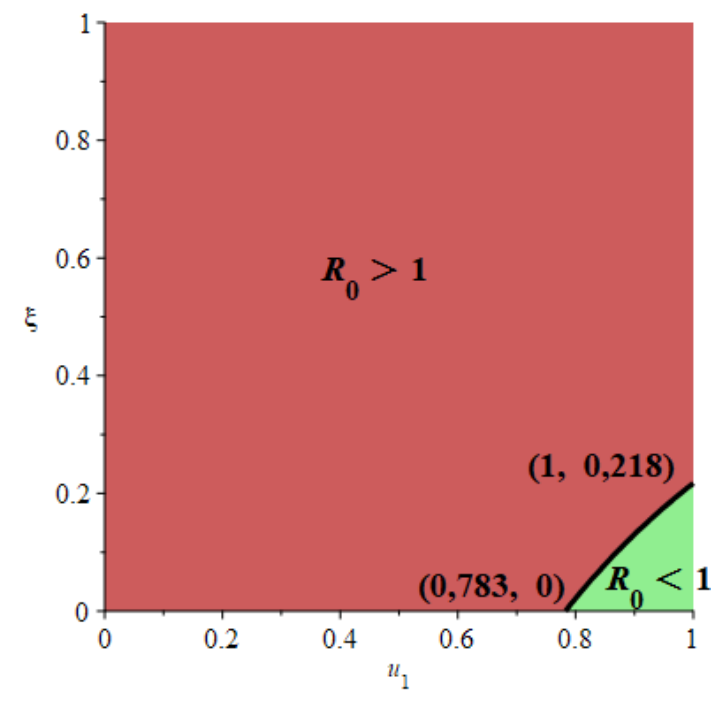

Figure 3. Sensitivity vaccination in newborns $\left(u_{1}\right)$ and vaccine efficacy $(\xi)$ to $\mathcal{R}_{0}$. 
In Figure 4, we assumed that only adults are vaccinated. The minimum vaccine cannot protect humans is $20,6 \%$ to lead a disease-free state. If a vaccine cannot protect, a minimum of $5,1 \%$ adults in the population must be vaccinated.

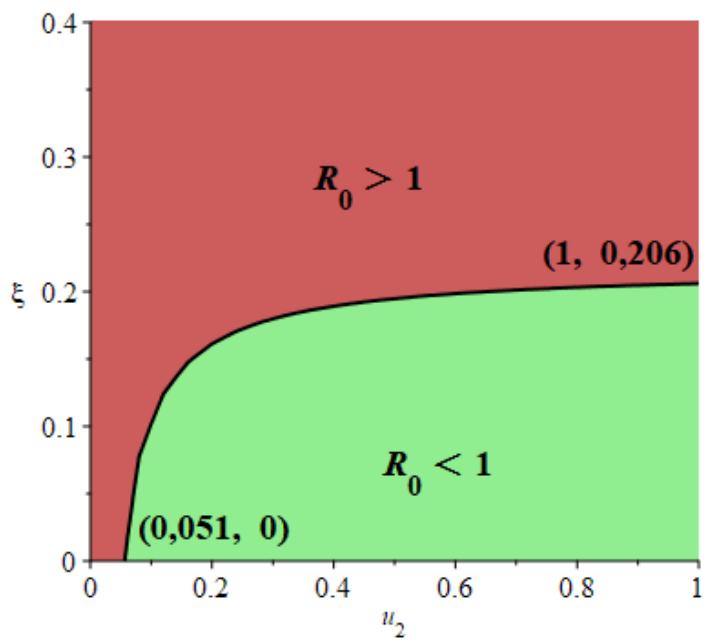

Figure 4. Sensitivity vaccination in adults $\left(u_{2}\right)$ and vaccine efficacy $(\xi)$ to $\mathcal{R}_{0}$.

4.2 Simulation of the autonomous system Table 2 . contains the initial values that used in the autonomous simulation.

Table 2. Initial Values of The Model

\begin{tabular}{cc}
\hline Variable state & Initial Value \\
$N$ & 1000 \\
$S$ & 650 \\
$V$ & 0 \\
$E$ & 200 \\
$L$ & 100 \\
$I$ & 150 \\
\hline
\end{tabular}

In the autonomic system, the effects of $u_{1}$ and $u_{2}$ are examined on infectious individuals during disease-free. We used three vaccination strategies: vaccination of newborns, adults, and both by selecting scenarios, as shown in table 3.

Table 3. Scenario $u_{1}$ and $u_{2}$ to $\mathcal{R}_{0}$ on disease-free.

\begin{tabular}{cc}
\hline Scenario $\boldsymbol{u}_{\mathbf{1}}$ and $\boldsymbol{u}_{\mathbf{2}}$ & $\boldsymbol{\mathcal { R }}_{\mathbf{0}}$ \\
$u_{1}=1, u_{2}=0$ & 0,92 \\
$u_{1}=0,5, u_{2}=0,5$ & 0,97 \\
$u_{1}=0, u_{2}=1$ & 0,97 \\
\hline
\end{tabular}

From Figure 5, we can see that the highest infectious population occurs when the vaccine is only given to all newborns. When $50 \%$ of newborns and adults were vaccinated, there was a significant reduction in the number of infectious individuals. Furthermore, when only adults got the vaccine, the number of infectious individuals was lower than the other two strategies.

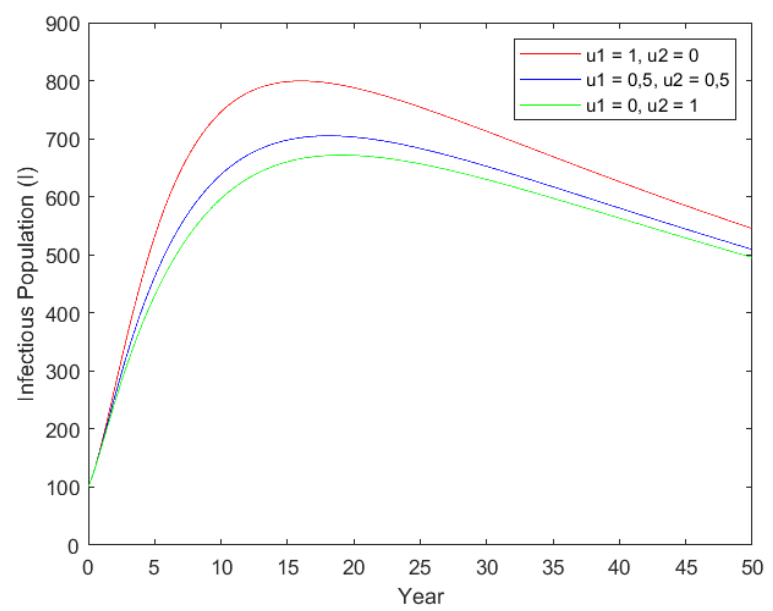

Figure 5. The autonomous system to disease-free.

In the second scenario, we used vaccination to newborns and adults during the disease-free and endemic conditions. The scenarios are shown in Table 4.

Table 4. Scenario $u_{1}$ and $u_{2}$ to $\mathcal{R}_{0}$ on disease-free and endemic condition.

\begin{tabular}{cc}
\hline Scenario $\boldsymbol{u}_{\mathbf{1}}$ and $\boldsymbol{u}_{\mathbf{2}}$ & $\mathcal{R}_{\mathbf{0}}$ \\
$u_{1}=0,1, u_{2}=0,8$ & 0,98 \\
$u_{1}=0,8, u_{2}=0,1$ & 1,01 \\
\hline
\end{tabular}

Table 4 shows that when $u_{1}$ and $u_{2}$ are 0,1 and 0,8 , then $\mathcal{R}_{0}<1$ is obtained, which means that the condition will be disease-free. On the other hand, when we use the proportion of $u_{1}$ and $u_{2}$ are 0,8 and 0,1 , we obtained $\mathcal{R}_{0}>1$, which means the condition will be endemic. Using this scenario, we get the autonomous system in Figure 6.

In Figure 6., it can be seen that the number of infectious populations will be reduced if the proportion of vaccination to adults is greater than that of newborns. 
Based on this, it seems that vaccination in adults is more effective than in newborns.

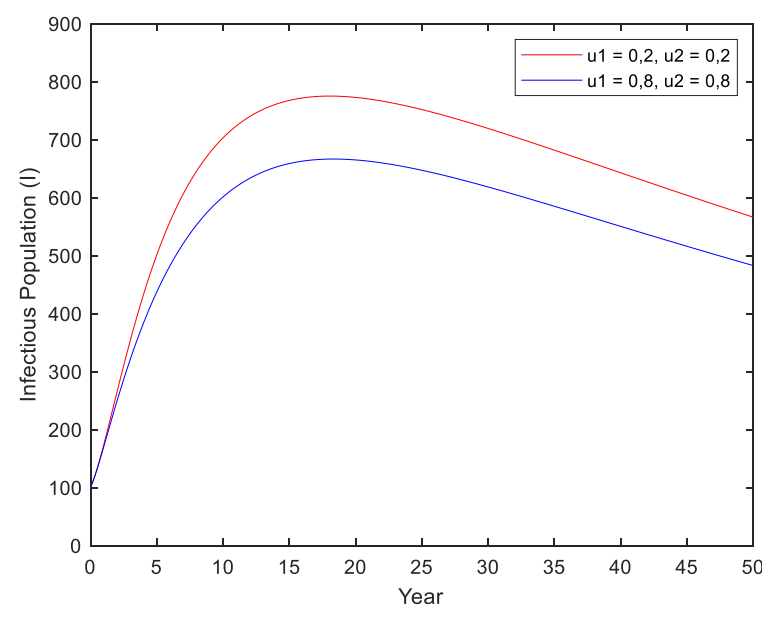

Figure 6. The autonomous system to disease-free and endemic.

\section{CONCLUSIONS AND SUGGESTIONS}

In this paper, we construct a mathematical model of TB with vaccination in controlling the spread of TB. Using the model, we analyzed the effect of vaccination on newborns and adults to reduce TB cases. From the numerical experiment, we can conclude that the lower the percentage of vaccines that cannot protect a human, the more likely it is to be disease-free. If the percentage vaccine could not protect humans $(\xi)$ is $20 \%$, more than $97,8 \%$ of newborns or $65 \%$ of adults in the population must be vaccinated. Furthermore, vaccination in adults is more effective than in newborns. Vaccination in adults can reduce the infectious population $(I)$ more than vaccination to newborns.

\section{ACKNOWLEDGMENTS}

This research was financially supported by Universitas Lampung, with Penelitian Dosen Pemula (PDP) research grant scheme 2020.

\section{REFERENCES}

Brauer, F., \& Castillo-ChavezCarlos. (2012). Mathematical models in population biology and epidemiology (2nd Ed). Springer.

Centers for Disease Control and Prevention (CDC). (2020). Latent TB infection and $T B$ disease. https://www.cdc.gov/tb/topic/basic s/tbinfectiondisease.htm

Choi, S., Jung, E., \& Lee, S. M. (2015). Optimal intervention strategy for prevention tuberculosis using a smoking-tuberculosis model. Journal of Theoretical Biology, 380. https://doi.org/10.1016/j.jtbi.2015. 05.022

Department of Health and Human Services. (2016). Management, control and prevention of tuberculosis. Melbourne Department of Health and Human Services.

Diekmann, O., Heesterbeek, J. A. P., \& Roberts, M. G. (2010). The construction of next-generation matrices for compartmental epidemic models. Journal of the Royal Society Interface, 7(47). https://doi.org/10.1098/rsif.2009.0 386

Grange, J. M., Brunet, L. R., \& Rieder, H. L. (2011). Immune protection against tuberculosis - When is immunotherapy preferable to vaccination? Tuberculosis, 91(2), 179-185.

https://doi.org/10.1016/j.tube.2010 .12 .004

Hethcote, H. W. (2000). Mathematics of infectious diseases. SIAM Review, 42(4), 599-653. https://doi.org/10.1137/S00361445 00371907

Houben, R. M. G. J., \& Dodd, P. J. (2016). The Global Burden of Latent Tuberculosis Infection: A Re-estimation Using Mathematical Modelling. PLoS Medicine, 13(10). https://doi.org/10.1371/journal.pm ed.1002152

Kim, S., de los Reyes, A. A., \& Jung, E. (2018). Mathematical model and intervention strategies for mitigating 
tuberculosis in the Philippines. Journal of Theoretical Biology, 443. https://doi.org/10.1016/j.jtbi.2018. 01.026

Liu, S., Li, Y., Bi, Y., \& Huang, Q. (2017). Mixed vaccination strategy for the control of tuberculosis: A case study in China. Mathematical Biosciences and Engineering, 14(3). https://doi.org/10.3934/mbe.20170 39

Mishra, B. K., \& Srivastava, J. (2014). Mathematical model on pulmonary and multidrug-resistant tuberculosis patients with vaccination. Journal of the Egyptian Mathematical Society, 22(2).

https://doi.org/10.1016/j.joems.201 3.07.006

Nguipdop-Djomo, P., Heldal, E., Rodrigues, L. C., Abubakar, I., \& Mangtani, P. (2016). Duration of BCG protection against tuberculosis and change in effectiveness with time since vaccination in norway: A retrospective population-based cohort study. THE LANCET Infectious Diseases, 16(12), 219-226. https://doi.org/https://doi.org/10.1 016/S1473-3099(15)00400-4

Nyabadza, F., \& Kgosimore, M. (2012). Modeling the Dynamics of Tuberculosis Transmission in Children and Adults. Journal of Mathematics and Statistics, 8(2). https://doi.org/10.3844/jmssp.2012 .229 .240

Petruccioli, E., Scriba, T. J., Petrone, L., Hatherill, M., Cirillo, D. M., Joosten, S. A., Ottenhoff, T. H., Denkinger, C. M., \& Goletti, D. (2016). Correlates of tuberculosis risk: Predictive biomarkers for progression to active tuberculosis. In European Respiratory Journal (Vol. 48, Issue 6). https://doi.org/10.1183/13993003. 01012-2016

Van Den Driessche, P., \& Watmough, J. (2002). Reproduction numbers and sub-threshold endemic equilibria for compartmental models of disease transmission. Mathematical Biosciences, 180(1-2). https://doi.org/10.1016/S00255564(02)00108-6

World Health Organization (WHO). (2019). Tuberculosis. https://www.who.int/newsroom/factsheets/detail/tuberculosis

Yang, Y., Tang, S., Ren, X., Zhao, H., \& Guo, C. (2016). Global stability and optimal control for a tuberculosis model with vaccination and treatment. Discrete and Continuous Dynamical Systems Series B, 21(3). https://doi.org/10.3934/dcdsb.2016 .21 .1009 
Desimal, 4 (1), 2021 - 56

S. L. Chasanah, P. Ferdias, D. E. Nurvazly 